\title{
Electron tomography based on highly limited data using a neural network reconstruction technique
}

\author{
Eva Bladt $^{\mathrm{a}, 1}$, Daniël M. Pelt ${ }^{\mathrm{b}, 1}$, Sara Bals ${ }^{\mathrm{a}, *}$, Kees Joost Batenburg ${ }^{\mathrm{b}, \mathrm{c}, \mathrm{d}}$ \\ a Electron Microscopy for Materials Research (EMAT), University of Antwerp, Groenenborgerlaan 171, B-2020 Antwerp, Belgium \\ ${ }^{\mathrm{b}}$ CWI, Science Park 123, 1098 XG Amsterdam, The Netherlands \\ c Mathematical Institute, Leiden University, Niels Bohrweg 1, 2333 CA Leiden, The Netherlands \\ d iMinds-Visionlab, University of Antwerp, Universiteitsplein 1, B-2610 Wilrijk, Belgium
}

\section{A R T I C L E I N F O}

\section{Article history:}

Received 20 January 2015

Received in revised form

20 May 2015

Accepted 7 July 2015

Available online 10 July 2015

Keywords:

Electron tomography

Neural networks

Reconstruction algorithm

Gold nanostructures

\begin{abstract}
A B S T R A C T
Gold nanoparticles are studied extensively due to their unique optical and catalytical properties. Their exact shape determines the properties and thereby the possible applications. Electron tomography is therefore often used to examine the three-dimensional (3D) shape of nanoparticles. However, since the acquisition of the experimental tilt series and the $3 \mathrm{D}$ reconstructions are very time consuming, it is difficult to obtain statistical results concerning the 3D shape of nanoparticles. Here, we propose a new approach for electron tomography that is based on artificial neural networks. The use of a new reconstruction approach enables us to reduce the number of projection images with a factor of 5 or more. The decrease in acquisition time of the tilt series and use of an efficient reconstruction algorithm allows us to examine a large amount of nanoparticles in order to retrieve statistical results concerning the 3D shape.
\end{abstract}

(c) 2015 Elsevier B.V. All rights reserved.

\section{Introduction}

Gold nanoparticles (NPs) have truly unique electronic, optical as well as catalytic properties, rendering them ideal for numerous applications in fields as diverse as photovoltaics, optoelectronics and biomedicine [1-4]. Furthermore, gold NPs can be prepared with almost any desired shape. Crucial to their application, however, is their exact structure, and specifically their anisotropy as well as the surface facets they expose. Currently, it is empirically understood how particle size and shape may be controlled during synthesis [5-8]. Although transmission electron microscopy (TEM) has become a routine tool to investigate e.g. particle size, (atomic) structure and shape, increasingly advanced TEM is required for a more in-depth characterisation. For example, the surface facets of Au nanorods have a major influence on crucial effects such as reactivity and ligand adsorption and there has been controversy regarding facet indexing [9-11]. Indeed, TEM images are only twodimensional (2D) projections of three-dimensional (3D) objects. To overcome this problem, 3D electron microscopy, or "electron tomography" was developed [12,13]. In 2003, Paul Midgley and coworkers demonstrated the potential of the technique in materials

\footnotetext{
* Corresponding author.

E-mail address: sara.bals@uantwerpen.be (S. Bals).

${ }^{1}$ E.B. and D.M.P. contributed equally.
}

science based on high angle annular dark field scanning transmission electron (HAADF-STEM) microscopy $[14,15]$. Since then, different electron microscopy modes have been combined successfully with tomography, leading to a broad variety of 3D structural and compositional information at the nanoscale [16-21]. Very often, electron tomography is used to determine the size and shape of the particles and nowadays, 3D reconstructions can even be obtained with a resolution at the atomic level [22,23]. Although these investigations provide very precise information on the NP morphology, both the acquisition of tilt series as well as the $3 \mathrm{D}$ reconstruction is very time consuming and it is consequently not straightforward to acquire results in 3D that are statistically relevant, which is a major drawback e.g. when using electron tomography to optimize the synthesis of NPs. This problem will be even more essential for anisotropic NPs that are currently receiving a lot of attention because of the increased flexibility they provide to tune the final (optical) properties [24-26]. Since the optimization of the production of NPs with a specific shape would largely benefit from statistical 3D results with a nanometer resolution, one of the emerging challenges in the field of electron tomography is to increase the throughput of 3D reconstructions of NPs. At the same time, the quality of the reconstructions should be maintained and should enable one to obtain reliable and quantitative results concerning parameters such as particle size and surface morphology.

In this paper, we will determine the $3 \mathrm{D}$ shape and size of a 
large set of anisotropic Au NPs. We will make effective use of a new approach for electron tomographic reconstructions that is based on artificial neural networks. The neural network filtered backprojection method (NN-FBP) is a recently developed reconstruction technique that has been applied successfully to X-ray tomography [27]; however the implementation for electron tomography is completely new. The method that we propose will enable us to reduce the number of necessary projection images for a $3 \mathrm{D}$ reconstruction by a factor of 5 or more. In this manner, the acquisition time and time that is necessary for a 3D reconstruction is significantly reduced, enabling 3D results that are of statistical relevance.

\section{Neural network filtered backprojection method}

The sample that was investigated contains Au NPs yielding different morphologies: nanorods, nanotriangles, nanoprisms and nanospheres. An HAADF-STEM overview image of the sample is provided in Fig. 1a. Although this image only corresponds to a $2 \mathrm{D}$ projection of a set of 3D objects, it is already clear that different morphologies occur. In conventional electron tomography, a large set of $2 \mathrm{D}$ projection images is acquired from the same region of interest over a large tilt range with a tilt increment of typically $1^{\circ}$ or $2^{\circ}$. As all the investigated nanoparticles have a thickness below $100 \mathrm{~nm}$, the projection requirement for tomography is satisfied $[14,28]$. Once this so-called "tilt series" is aligned, the images serve as an input for a mathematical algorithm that enables one to reconstruct the original 3D structure. Very often, the 3D reconstruction is performed using the "Weighted Backprojection" algorithm (also known as Filtered Backprojection) or using the "Simultaneously Iterative Reconstruction Technique" (SIRT). The outcome of this procedure for the different NPs in Fig. 1a is visualized in Fig. 1b. The reconstructions are calculated using the SIRT algorithm and are based on a series of 151 images, acquired over a tilt range of $\pm 75^{\circ}$. Since the quality of 3D reconstructions based on the conventional approach is predominantly determined by the number of projection images [29-31], these experiments are very time-consuming and require sufficient measurement time at the TEM.
The key to increasing the image quality if only a small number of $2 \mathrm{D}$ projections are available, is the effective use of prior knowledge in the reconstruction. By exploiting rather generic features of the particles, without assuming a specific shape or morphology, this additional knowledge is used to compute a particle shape that better approximates the true morphology. Various algorithms involving prior knowledge are currently in use in electron tomography (e.g. the DART algorithm for discrete tomography [32] and multiple methods for Total Variation Minimization [33]), where the particular prior knowledge is encoded by the user and various parameters have to be set. These priorknowledge based methods are typically very time-consuming, which limits the throughput of 3D reconstructions that can be achieved by using them for reconstruction. Furthermore, implementing these methods can be difficult and time-consuming as well, since they rely on advanced mathematics. In this paper, we propose an alternative approach called Neural Network Filtered Backprojection (NN-FBP) that was first described in [27], which can effectively exploit sample characteristics to improve reconstruction quality, while still being highly computationally efficient. Here, we apply this new technique for the first time to electron tomography data. The application of NN-FBP to electron tomography consists of two phases: (i) a learning phase, in which full tilt series and their corresponding reconstructions are used to calibrate the reconstruction algorithm and (ii) a reconstruction phase, in which large batches of limited tilt series (i.e. using fewer projections) are rapidly reconstructed. A schematic overview the NN-FBP method is given in Fig. 2. In the next subsections, we will first briefly explain how the reconstructions are formed in the reconstruction phase, followed by an overview of how the calibration is performed in the learning phase.

\subsection{Reconstruction phase}

Reconstructions obtained by standard Weighted Backprojection are commonly plagued by a range of reconstruction artefacts when reconstructing from a limited tilt range and few projection angles. Streaks can be observed due to the limited number of projections, and the limited angular range leads to elongation and blurring in the Z-direction. In [27], it was found that strong improvements on
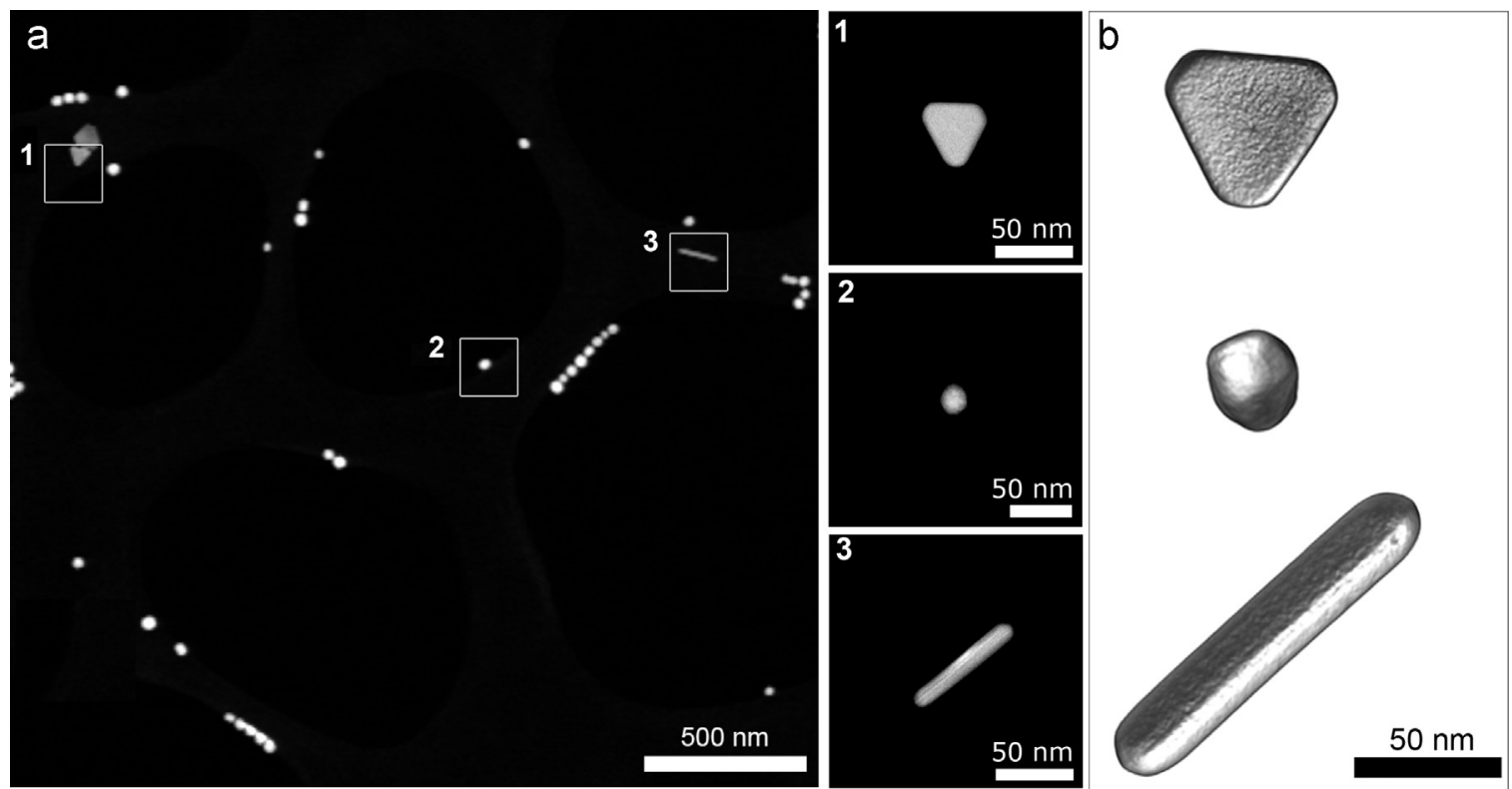

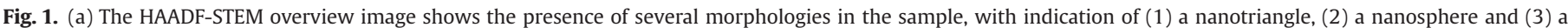
nanorod. (b) 3D volume renderings of the corresponding nanoparticles are presented. 


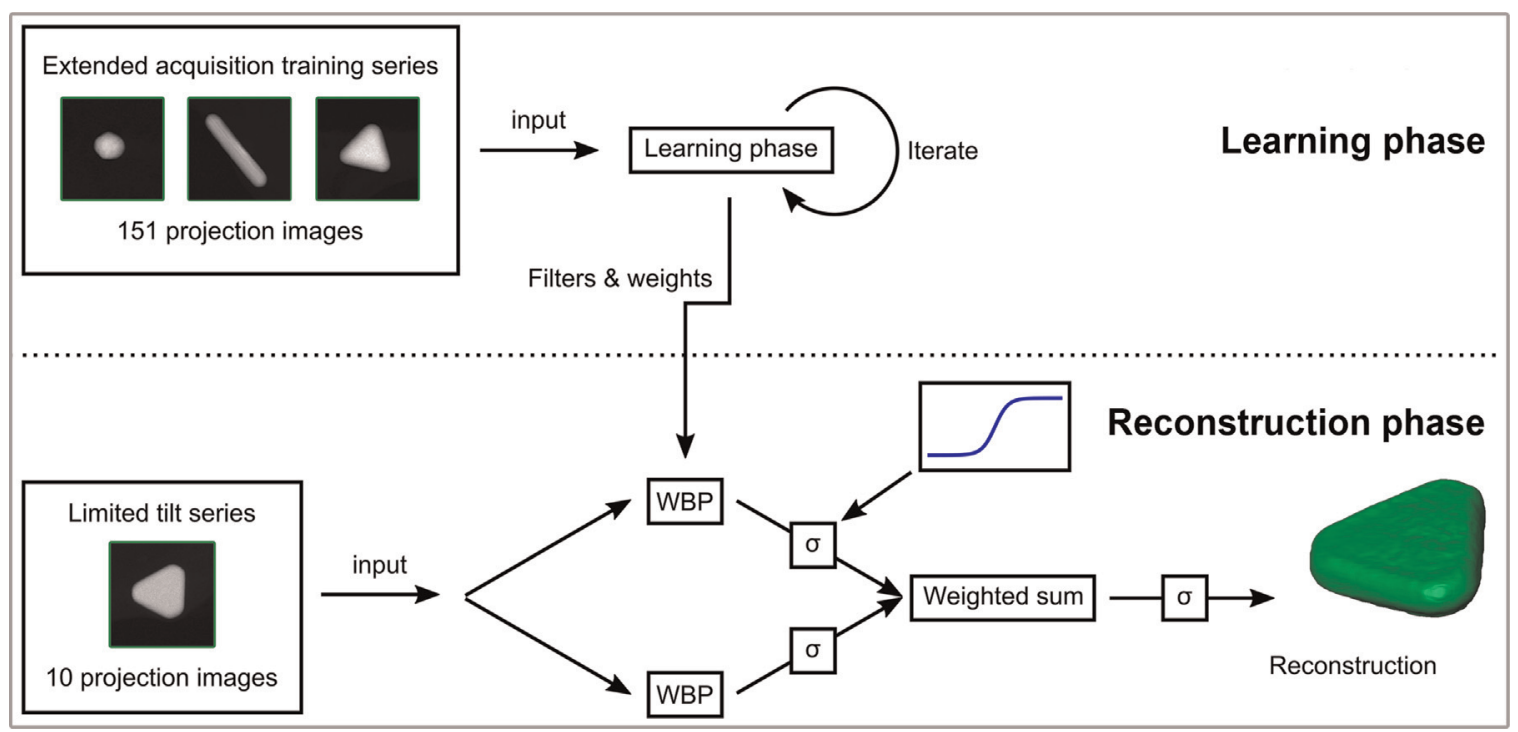

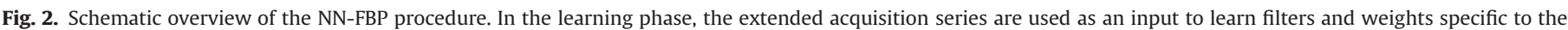

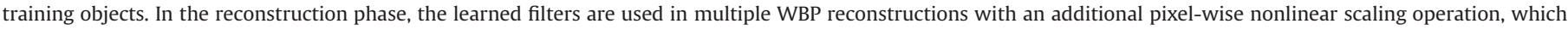
are combined to obtain a single reconstruction of a limited tilt series.

the reconstruction quality from limited data can be obtained by combining a small number (e.g. 2 or 4 ) of WBP reconstructions, each obtained using a different filter.

In the reconstruction phase, the NN-FBP algorithm computes a reconstructed volume from limited projection data by combining multiple WBP reconstructions with different filters into a single reconstruction. A key ingredient of the algorithm is the application of a pixel-wise nonlinear scaling operation to each of the WBP images. Following this operation, the images are combined by taking a weighted sum of the scaled WBP images. As a final step, another nonlinear scaling operation is applied to this combined image (see reconstruction phase in Fig. 2).

Note that without these nonlinear scaling operations, the final reconstruction can also be obtained by first creating a weighted sum of the different filters, and performing a Weighted Backprojection with the resulting filter, as the WBP algorithm is a linear method with respect to the used filter. Because of this, such a method will not be able to produce more accurate reconstructions than standard Weighted Backprojection with an appropriately chosen filter. Also, because of the nonlinear scaling operation, it is not possible to directly compare the filters of the NN-FBP method with standard filters for WBP.

By using the nonlinear scaling operation, the NN-FBP algorithm is able to reduce the artefacts that are usually present in standard Weighted Backprojection reconstructions when only a small number of projections are available. An example image with standard Weighted Backprojection, a linear combination of two Weighted Backprojections, and a combination of two Weighted Backprojections with nonlinear scaling is shown in Fig. 3. As expected, the figure shows that the linear combination is identical to a single Weighted Backprojection reconstruction, while the combination with nonlinear scaling is significantly more accurate.

\subsection{Learning phase}

The question remains how the different filters and weights have to be chosen, such that the method produces accurate reconstructions. In [27], it is shown that ideas from artificial neural network theory can be used to find good filters and weights. Specifically, filters and weights can be learned by the NN-FBP method in a separate learning phase, in which the method is presented with high-quality reconstructions of a set of training objects. In artificial neural network theory, this technique is called supervised learning. In the learning phase, the filters and weights are iteratively adjusted until the NNFBP reconstructions match the presented high-quality reconstructions. Afterwards, the trained filters and weights can be used to accurately reconstruct objects that are similar to the ones used for training, using only a limited number of projections. The angle distribution of the limited number of projections has to be specified during the learning phase, and the learned filters and weights will be specific to the chosen distribution. To reduce the influence of the specific angles that are chosen, NN-FBP uses angle-independent
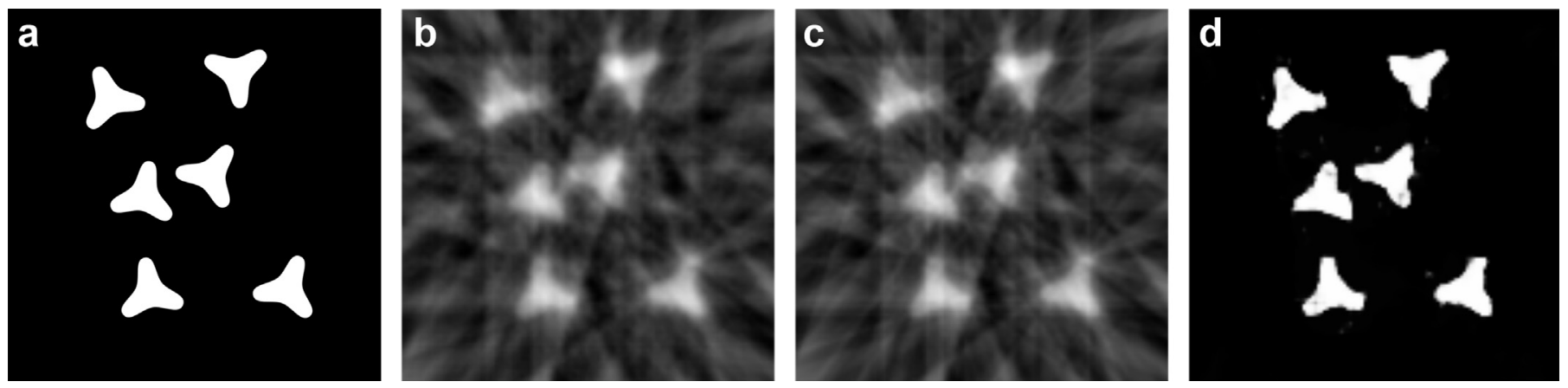

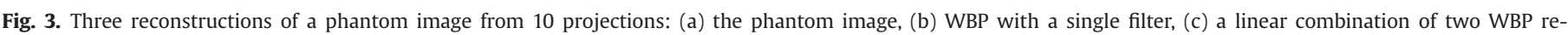

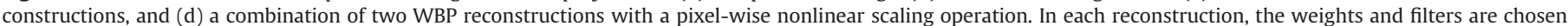
such that the mean squared error with the phantom image is minimized. 
filters, i.e. the same filters are used for each projection. An important requirement of the NN-FBP method is that the reconstructed objects should consist only of materials that were also present in the training objects. When this requirement is satisfied, the NN-FBP method is able to produce accurate reconstructions, even for objects with different shapes and/or sizes as the training objects. A schematic overview of both the learning phase and subsequent reconstruction of the NN-FBP method is given in Fig. 2.

As opposed to previous advanced reconstruction methods, specific prior knowledge is not explicitly used in the NN-FBP method. Instead, the method learns to exploit certain characteristics of the training objects by adjusting the filters and weights appropriately. Because the exploited characteristics are learned automatically by the method, it has a broader applicability than previous advanced 3D reconstruction methods. Also, since NN-FBP is based on the efficient Weighted Backprojection algorithm, it is computationally efficient as well, enabling high throughput of 3D reconstructions. An additional advantage is that existing implementations of the Weighted Backprojection algorithm can be used to easily implement the NN-FBP method. A final advantage is that it is possible to include the segmentation step in the NN-FBP method by using segmented high-quality reconstructions of the training objects in the learning phase. In this case, the NN-FBP method will reconstruct objects with voxel values that are very close to their segmented value, and the final segmentation can be performed by simple rounding to the nearest segmented value. This removes the need for manual segmentation, which can be problematic for other methods when only a limited set of projections is available.

\section{Results}

\subsection{Qualitative results}

In a first experiment, tilt series of a nanosphere, a nanorod and a nanotriangle are acquired over an angular tilt range of $\pm 75^{\circ}$ with a tilt increment of $1^{\circ}$. These three series are used as training series, resulting in a set of filters that will be used during the NNFBP approach. The resulting NN-FBP algorithm is applied to a limited tilt series that was acquired from a different nanotriangle. Although only 10 projection images obtained over a range of $\pm 75^{\circ}$ are used during the NN-FBP reconstruction, it needs to be pointed out that we also acquired an extended series of 151 projection images. The SIRT reconstruction of the extended dataset was used as ground truth, in order to evaluate the NN-FBP outcome. Fig. 4a presents a volume rendering of this full range SIRT reconstruction. In all experiments, we used 200 iterations for the SIRT reconstructions, which was empirically verified to produce accurate reconstructions. The result of the NN-FBP algorithm is shown in Fig. $4 \mathrm{~b}$. It must be stressed that in this case only 10 projection images were used. It can be seen that the $3 \mathrm{D}$ volume visualisation of the NN-FBP reconstruction is in very good agreement with the SIRT reconstruction of the full data series. The top and side facet can clearly be distinguished in the corresponding orthoslices in Fig. $4 \mathrm{e}, \mathrm{i}, \mathrm{m}$ and $\mathrm{f}, \mathrm{j}, \mathrm{n}$. On the other hand, when comparing the SIRT reconstruction based on the extended series with the SIRT reconstruction based on 10 projection images (Fig. 4c, g, k and o), it can be seen that the faceted shape is less pronounced. In the WBP reconstruction applied on 10 projection images (Fig. 4d, h, 1
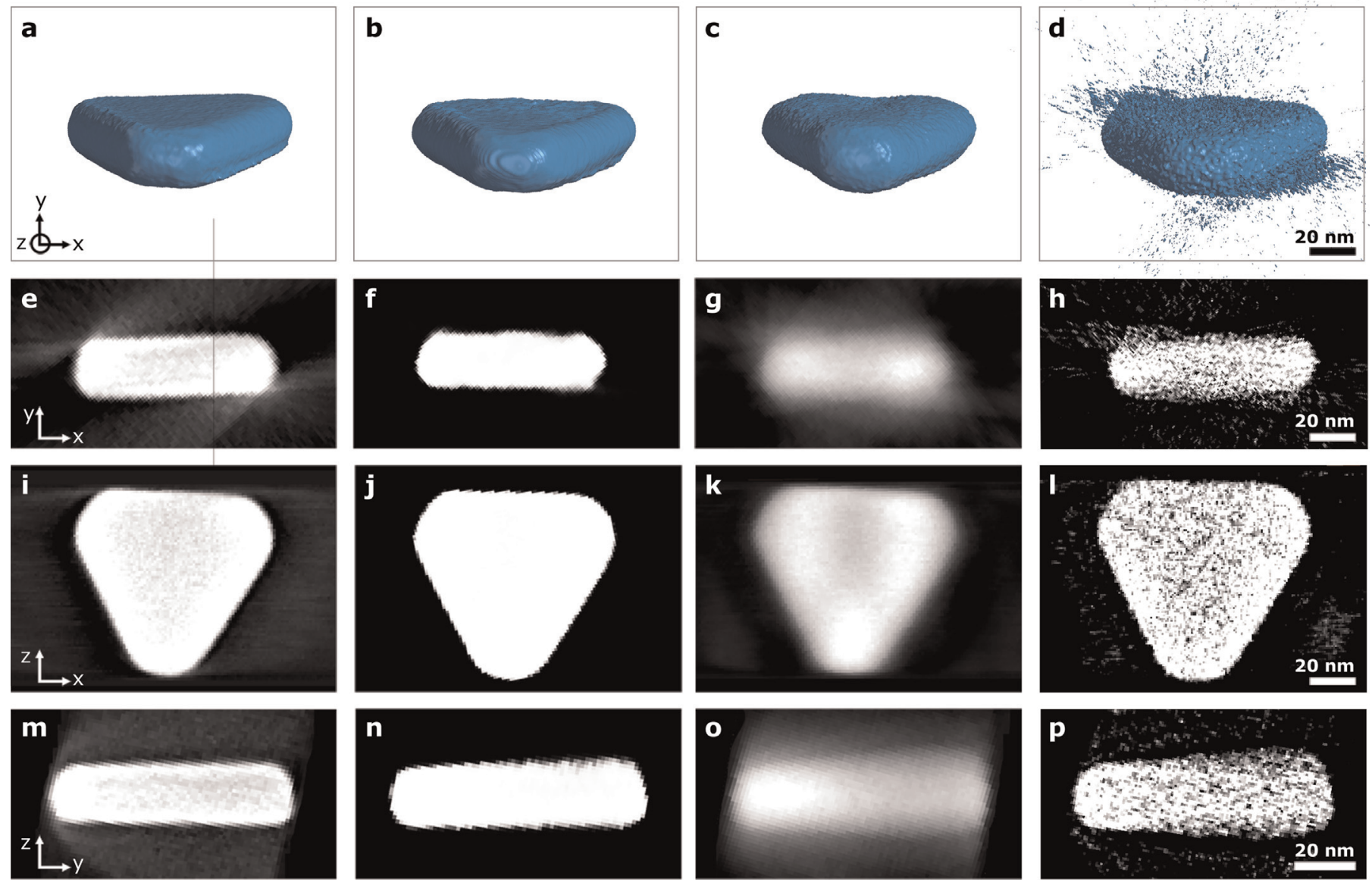

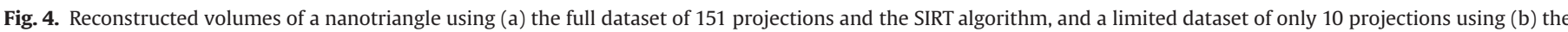

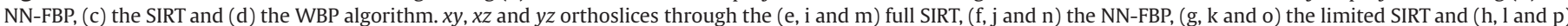
the limited WBP reconstructions of the nanotriangle. 

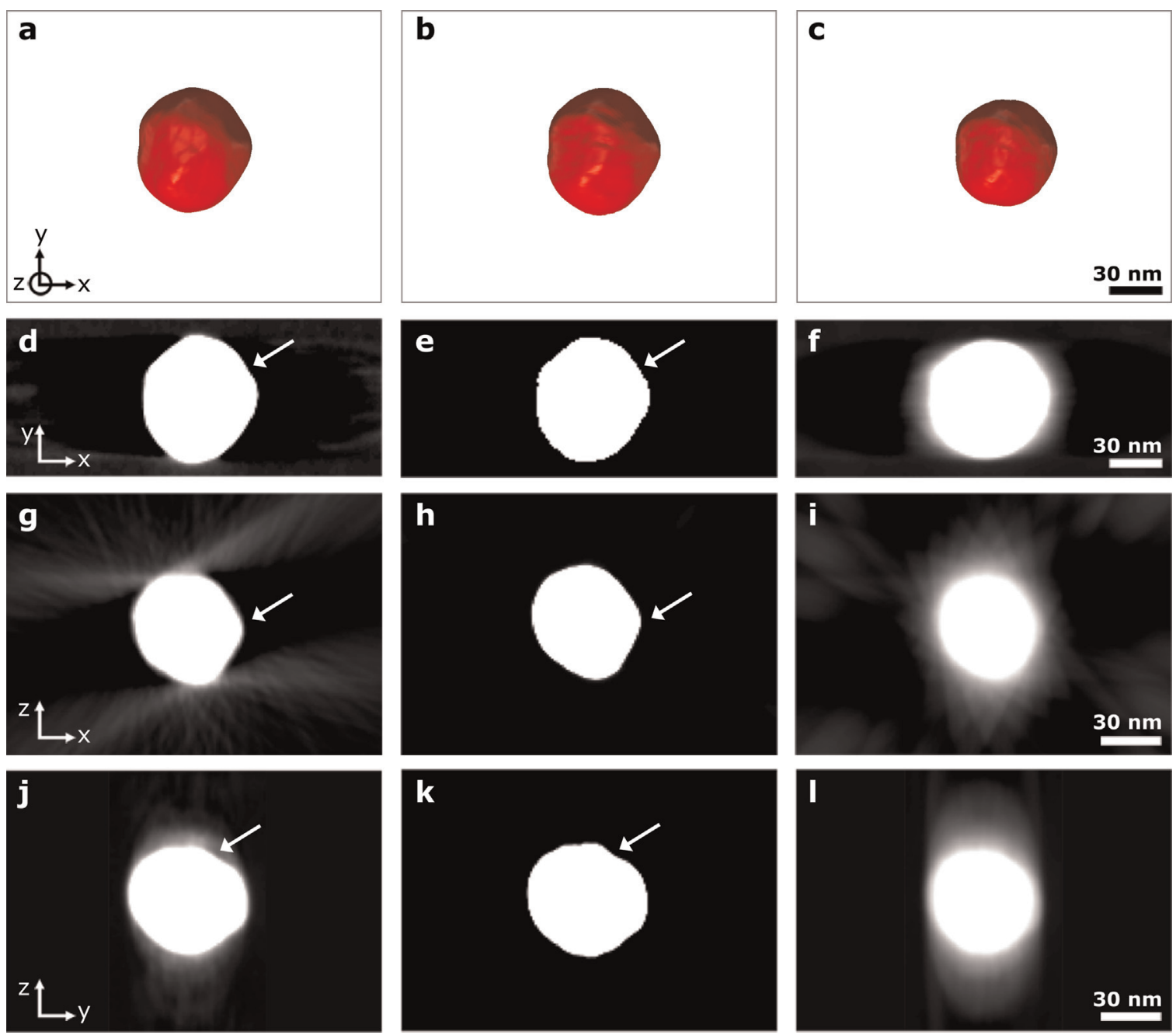

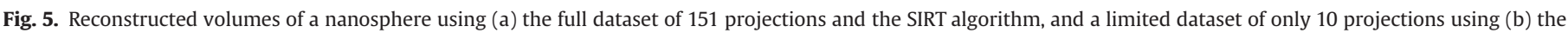

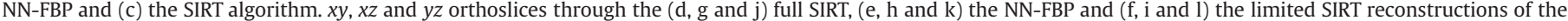

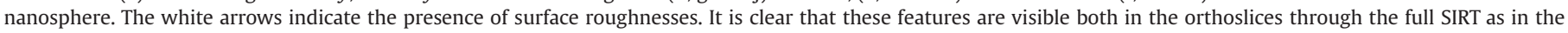
orthoslices through the NN-FBP reconstruction; however, in the limited SIRT reconstruction they are not detectable.

and p), severe noise and streaking artefacts can be distinguished. These artefacts can be prohibitive for further analysis of the scanned object, such as volume or shape calculations. Therefore, the WBP reconstruction will be left out in the further analysis. The benefits of NN-FBP become obvious; the number of images required for a $3 \mathrm{D}$ reconstruction using NN-FBP is reduced by a factor of 15 , but the quality is comparable to a reconstruction based on a full data series with a tilt increment of $1^{\circ}$.

In Figs. 5 and S1, results for a nanosphere and a nanorod are presented, respectively. Here, the training of the filters was again obtained by 3 training series. For the nanosphere, extended series of the nanorod and both nanotriangles were used. The training step for the nanorod was performed by the extended series of the nanosphere and both nanotriangles. These nanostructures yield fewer facets and as a consequence, the general morphology as visualised in Figs. 5b, c and S1b, c appears to be better preserved when using only 10 projections. However, missing wedge artefacts can be clearly seen in the orthoslices presented in Figs. $5 \mathrm{f}, \mathrm{i}, \mathrm{l}$ and S1f, i, l. Because of such artefacts, some features of the morphology indicated by white arrows in both the orthoslices through the full SIRT reconstruction (Fig. $5 \mathrm{~d}, \mathrm{~g}$ and $\mathrm{j}$ ) and the NN-FBP reconstruction (Fig. 5e, h and $\mathrm{k}$ ) are not clearly visible in the orthoslices through the limited SIRT reconstruction (Fig. 5f, i and 1).

\subsection{Quantitative results}

As a quantitative measure, a difference reconstruction for the nanosphere is constructed by substracting the SIRT (Fig. 6a) and NN-FBP reconstructions based on 10 projection images (Fig. 6b) from the full SIRT reconstruction of the nanosphere. The threshold value for the full SIRT reconstruction is obtained from the histogram. The histogram of the limited SIRT reconstruction, however, is lagerly influenced by the lack of projection images. In Fig. S2, comparisons are shown between the histograms of the full SIRT reconstruction and the limited SIRT reconstruction for each nanoparticle. Clearly, one would have trouble choosing correct threshold values on the basis of the limited SIRT histograms. Therefore, the same threshold value as the full SIRT reconstruction is used for the limited SIRT reconstructions. Since the NN-FBP reconstructions are already segmented, no threshold value is needed for them. Both from the visualisation in Fig. 6a, as well as the corresponding orthoslices through the difference reconstruction in Fig. 6c, the volume misinterpretation of the limited SIRT reconstruction is clearly detectable. The orthoslices through the limited SIRT difference reconstruction of the nanosphere show a thick white shell. Here, the larger amount of white pixels indicates a volume misinterpretation of $21.5 \%$ when using the SIRT algorithm on the dataset of only 10 projection images. From Fig. $6 \mathrm{~b}$ and 


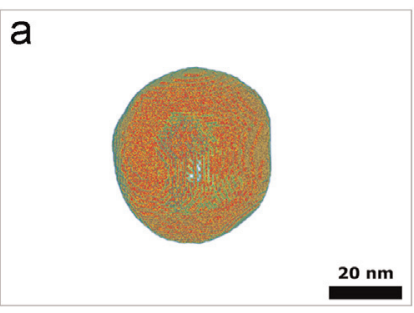

\section{b}
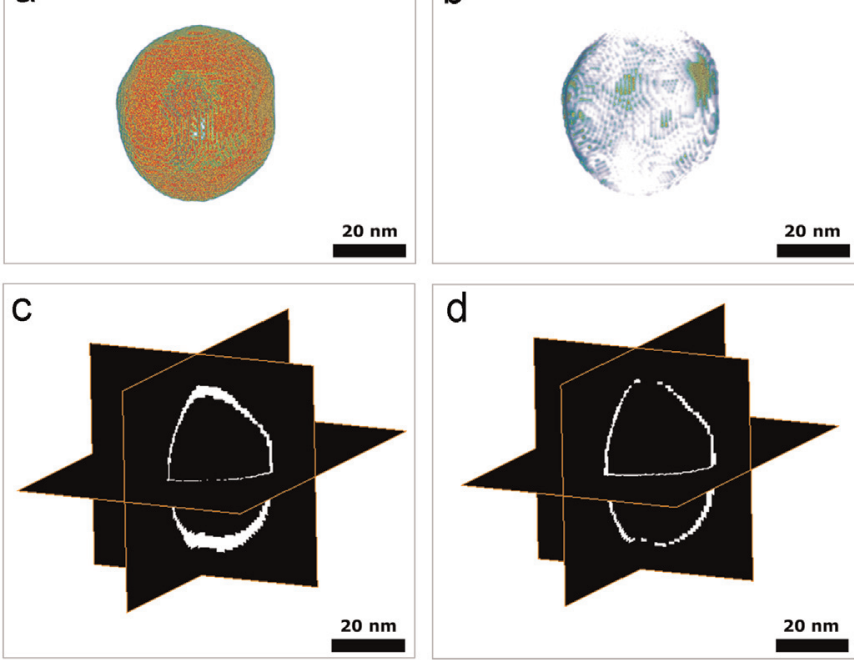

Fig. 6. Difference reconstructions of the nanosphere constructed by substracting (a) the SIRT and (b) NN-FBP reconstruction of 10 projection images from the full SIRT reconstruction representing the missing volume and its orthoslices (c) and (d), respectively. The volume misinterpretation for the NN-FBP reconstruction equals only $1.6 \%$, which is indicated by the fine shell of the difference reconstruction. The thicker shell present in the difference reconstruction of the limited SIRT equals a volume misinterpretation of $21.5 \%$.

its corresponding orthoslices in Fig. 6d, it is clear that the volume reconstructed with NN-FBP on 10 projection images is close to the actual volume. The NN-FBP reconstruction has only $1.6 \%$ of volume underestimation. For the nanorod (Fig. S3), the volume for SIRT applied to a limited dataset results in an underestimation of $13.1 \%$. The NN-FBP reconstruction leads to a misinterpretation of only $2.3 \%$. For the nanotriangle, the volume misinterpretation for the limited SIRT reconstruction equals $2.7 \%$. When reconstructing the 10 projection dataset with the NN-FBP algorithm, the volume misinterpretation equals $2.4 \%$. For the nanotriangle, the volume misinterpretation of the limited SIRT reconstruction is close to the misinterpretation of the NN-FBP reconstruction. In this case, however, the volume misinterpretation of the limited SIRT reconstruction gives a misleading result, due to a volume underestimation at the center of the nanotriangle and a volume overestimation at the tips of the nanotriangle. In general, the volume misinterpretation can be misleading due to the canceling out of overestimation and underestimation. Clearly, the evaluation of the quality of the reconstruction can not only be based on an inspection of the volume error. Therefore, the shape error is introduced, which corresponds to the number of voxels that are labelled differently in the segmentations of the limited data reconstructions in comparison to the full SIRT reconstruction. In this manner, both the local volume underestimation at the center as well as the volume overestimation at the tips is taken into account. For the nanotriangle, there is a $16.5 \%$ shape misinterpretation for the limited SIRT reconstruction (Fig. S4). The shape error for the NN-FBP reconstruction equals $7.5 \%$, which is clearly smaller in comparison to the shape error of the limited SIRT reconstruction. An extended investigation of the influence of the chosen threshold value on the shape error and volume error of the limited SIRT reconstructions is shown in Fig. S5. Note that from Fig. S5, one can conclude that the errors depend heavily on the chosen threshold value, showing the difficulties one would have when choosing a threshold value both optimizing shape and volume error for limited SIRT reconstructions.

\subsection{Statistical results}

In general it is difficult to obtain statistical results when applying electron tomography. As pointed out previously, the acquisition of tilt series for electron tomography is very time consuming and a large electron dose is required in the case of small tilt increments. The NN-FBP algorithm is therefore of great interest as it can be applied to reduce the acquisition time. In this manner a large set of nanostructures can be investigated in an efficient manner, leading to statistical results. Using the NN-FBP approach explained above, training was performed on a set of 20 nanoparticles, and a total of 71 nanospheres was investigated. The number of nanoparticles to train on was chosen empirically, such that there were both enough particles to use in the learning phase, and enough particles to obtain statistical results from. In Fig. 7, the distribution of the radii of these nanospheres is evaluated. In order to investigate the reliability of the NN-FBP approach, extended tilt series of 151 images were acquired for all particles. The outcome of

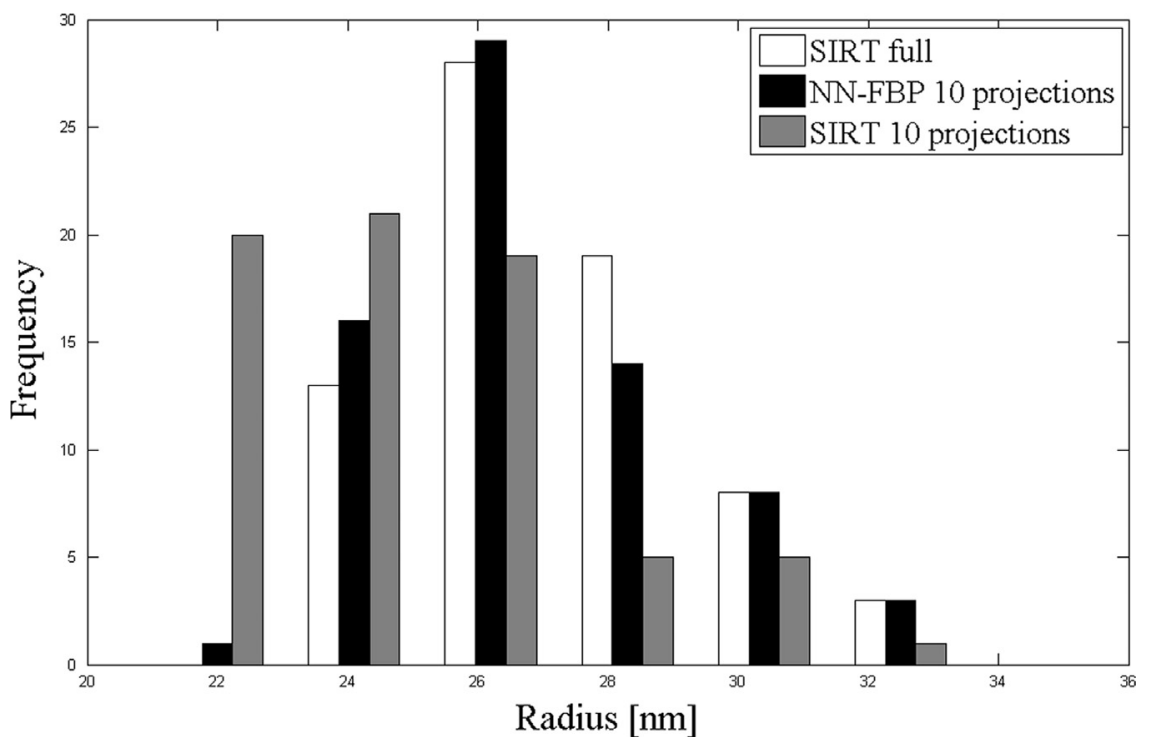

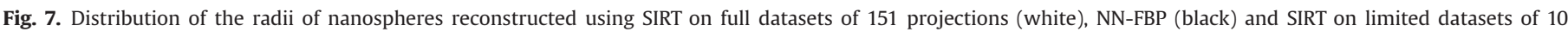

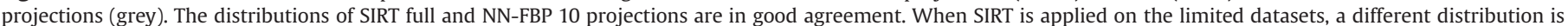
found due to the misinterpretation of the volume. 
the NN-FBP algorithm and the SIRT algorithm, using only 10 projections, is then compared to the measurements based on the SIRT reconstruction using 151 projections. The distribution indicated in grey in Fig. 7 presents the radii distribution for the nanospheres reconstructed using SIRT applied to limited datasets and clearly gives a different distribution in comparison to the radii distribution of the full SIRT reconstruction, which is presented in white. The average radius found in this manner equals $(24.1 \pm 0.59) \mathrm{nm}$, which is significantly smaller than the actual radius which equals $(27.1+0.25) \mathrm{nm}$, found through the full SIRT reconstructions. As the optical properties, such as the absorption cross section, are dependent on the shape and size of the nanoparticles, it is of key importance to retrieve the real nanoparticle morphology. A difference of a few nanometer can already influence the outcome of the optical response $[34,35]$. The radii distribution of the NN-FBP reconstruction (black), however, is in good agreement with the results extracted from the full SIRT data (white). The average radius of the NN-FBP reconstructed nanospheres equals $(26.8 \pm 0.29) \mathrm{nm}$. This value is in good agreement with the actual average radius and shows a clear overlap of the error bars. It is again clear that the SIRT algorithm can not provide reliable information when limited datasets are investigated. These results confirm the reliability of the NNFBP algorithm and demonstrate the possibility of combining electron tomography and statistical measurements.

\section{Conclusion}

We have shown that the NN-FBP reconstruction algorithm is able to yield electron tomography reconstructions based on highly limited data with a comparable quality to a reconstruction based on a full data series with a tilt increment of $1^{\circ}$. The decrease in acquisition time and the use of an efficient reconstruction method enables us to examine a broad range of nanostructures in a statistical manner. The NN-FBP algorithm also has promising prospects for the 3D investigation of beam sensitive samples, where only a limited amount of projection images need to be acquired.

\section{Supplementary information}

Reconstructed volumes of a nanorod, histograms of the SIRT reconstructions, difference reconstructions of the nanorod, representations of the shape misinterpretation of the nanotriangle and plots of the relative error in the shape and the volume of the different nanostructures.

\section{Acknowledgment}

S.B. acknowledges financial support from European Research Council (ERC Starting Grant \#335078-COLOURATOMS). E.B. gratefully acknowledges financial support by The Flemish Fund for Scientific Research (FWO Vlaanderen). D.M.P. and K.J.B. acknowledge financial support by The Netherlands Organisation for Scientific Research (NWO), Project number 639.072.005. The authors acknowledge COST Action MP1207 for networking support and the European Union under the Seventh Framework Program under a contract for an Integrated Infrastructure Initiative, Reference no. 312483-ESTEEM2 for financial support. We would like to thank Luis M. Liz-Marzán for provision of the samples.

\section{Appendix A. Supplementary information}

Supplementary data associated with this article can be found in the online version at http://dx.doi.org/10.1016/j.ultramic.2015.07.001.

\section{References}

[1] P. Zijlstra, J.W.M. Chon, M. Gu, Five-dimensional optical recording mediated by surface plasmons in gold nanorods, Nature 459 (2009) 410-413, http://dx.doi. org/10.1038/nature08053.

[2] P. Zijlstra, M. Orrit, Single metal nanoparticles: optical detection, spectroscopy and applications, Rep. Prog. Phys. 74 (2011) 106401., http://dx.doi.org/10.1088/ 0034-4885/74/10/106401.

[3] N.L. Rosi, C.A. Mirkin, Nanostructures in biodiagnostics, Chem. Rev. 105 (2005) 1547-1562, http://dx.doi.org/10.1021/cr030067f.

[4] R. Cao-Milán, L.M. Liz-Marzán, Gold nanoparticle conjugates: recent advances toward clinical applications, Expert Opin. Drug Deliv. 11 (2014) 741-752, http: //dx.doi.org/10.1517/17425247.2014.891582.

[5] J. Pérez-Juste, I. Pastoriza-Santos, L.M. Liz-Marzán, P. Mulvaney, Gold nanorods: synthesis, characterization and applications, Coord. Chem. Rev. 249 (2005) 1870-1901, http://dx.doi.org/10.1016/j.ccr.2005.01.030.

[6] M. Grzelczak, J. Pérez-Juste, P. Mulvaney, L.M. Liz-Marzán, Shape control in gold nanoparticle synthesis, Chem. Soc. Rev. 37 (2008) 1783-1791, http://dx. doi.org/10.1039/b711490g.

[7] A. Sánchez-Iglesias, I. Pastoriza-Santos, J. Pérez-Juste, B. Rodríguez-González, F J. García de Abajo, L.M. Liz-Marzán, Synthesis and optical properties of gold nanodecahedra with size control, Adv. Mater. 18 (2006) 2529-2534, http://dx. doi.org/10.1002/adma.200600475.

[8] A.R. Tao, S. Habas, P. Yang, Shape control of colloidal metal nanocrystals, Small 4 (2008) 310-325, http://dx.doi.org/10.1002/smll.200701295.

[9] C. Pecharromán, J. Pérez-Juste, G. Mata-Osoro, L. Liz-Marzán, P. Mulvaney, Redshift of surface plasmon modes of small gold rods due to their atomic roughness and end-cap geometry, Phys. Rev. B 77 (2008) 035418, http://dx. doi.org/10.1103/PhysRevB.77.035418.

[10] E. Carbó-Argibay, B. Rodríguez-González, S. Gómez-Graña, A. GuerreroMartínez, I. Pastoriza-Santos, J. Pérez-Juste, et al., The crystalline structure of gold nanorods revisited: evidence for higher-index lateral facets, Angew. Chem. Int. Ed. Engl. 49 (2010) 9397-9400, http://dx.doi.org/10.1002/ anie.201004910.

[11] H. Katz-Boon, C.J. Rossouw, M. Weyland, A.M. Funston, P. Mulvaney, J. Etheridge, Three-dimensional morphology and crystallography of gold nanorods, Nano Lett. 11 (2011) 273-278, http://dx.doi.org/10.1021/nl103726k.

[12] A.J. Koster, R. Grimm, D. Typke, R. Hegerl, A. Stoschek, J. Walz, et al., Perspectives of molecular and cellular electron tomography, J. Struct. Biol. 120 (1997) 276-308, http://dx.doi.org/10.1006/jsbi.1997.3933.

[13] J. Frank, Electron Tomography: Methods for Three-Dimensional Visualization of Structures in the Cell, Plenum Press, New York, London, 1992.

[14] P.A. Midgley, M. Weyland, 3D electron microscopy in the physical sciences: the development of Z-contrast and EFTEM tomography, Ultramicroscopy 96 (2003) 413-431, http://dx.doi.org/10.1016/S0304-3991(03)00105-0.

[15] P.A. Midgley, R.E. Dunin-Borkowski, Electron tomography and holography in materials science, Nat. Mater. 8 (2009) 271-280, http://dx.doi.org/10.1038/ NMAT2406.

[16] D. Wolf, A. Lubk, H. Lichte, H. Friedrich, Towards automated electron holographic tomography for 3D mapping of electrostatic potentials, Ultramicroscopy 110 (2010) 390-399, http://dx.doi.org/10.1016/j.ultramic.2009.12.015.

[17] M. Weyland, T.J.V. Yates, R.E. Dunin-Borkowski, L. Laffont, P.A. Midgley, Nanoscale analysis of three-dimensional structures by electron tomography, Scr. Mater. 55 (2006) 29-33, http://dx.doi.org/10.1016/j.scriptamat.2005.12.058.

[18] M. Bar Sadan, L. Houben, S.G. Wolf, A. Enyashin, G. Seifert, R. Tenne, et al., Toward atomic-scale bright-field electron tomography for the study of fullerene-like nanostructures, Nano Lett. 8 (2008) 891-896, http://dx.doi.org/ 10.1021/nl073149i.

[19] P.A. Midgley, S. Bals, Electron tomography, in: G. Van Tendeloo, D. Van Dyck, S.J. Pennycook (Eds.), Handbook of Nanoscopy, Wiley-VCH Verlag GmbH \& Co. KGaA, 2012, pp. 253-279, http://dx.doi.org/10.1002/9783527641864.ch7.

[20] B. Goris, S. Bals, W. Van den Broek, J. Verbeeck, G. Van Tendeloo, Exploring different inelastic projection mechanisms for electron tomography, Ultramicroscopy 111 (2011) 1262-1267, http://dx.doi.org/10.1016/j. ultramic.2011.02.007.

[21] A.J. Koster, U. Ziese, A.J. Verkleij, A.H. Janssen, K.P. de Jong, Three-dimensional transmission electron microscopy: a novel imaging and characterization technique with nanometer scale resolution for materials science, J. Phys. Chem. B 104 (2000) 9368-9370, http://dx.doi.org/10.1021/jp0015628.

[22] B. Goris, S. Bals, W. Van den Broek, E. Carbó-Argibay, S. Gómez-Graña, L.M. LizMarzán, et al., Atomic-scale determination of surface facets in gold nanorods, Nat. Mater. 11 (2012) 930-935, http://dx.doi.org/10.1038/nmat3462.

[23] S. Van Aert, K.J. Batenburg, M.D. Rossell, R. Erni, G. Van Tendeloo, Three-dimensional atomic imaging of crystalline nanoparticles, Nature 470 (2011) 374-377, http://dx.doi.org/10.1038/nature09741.

[24] S.C. Glotzer, M.J. Solomon, Anisotropy of building blocks and their assembly into complex structures, Nat. Mater. 6 (2007) 557-562, http://dx.doi.org/ $10.1038 /$ nmat1949.

[25] Z. Nie, A. Petukhova, E. Kumacheva, Properties and emerging applications of self-assembled structures made from inorganic nanoparticles, Nat. Nanotechnol. 5 (2010) 15-25, http://dx.doi.org/10.1038/nnano.2009.453.

[26] A. Guerrero-Martínez, M. Grzelczak, L.M. Liz-Marzán, Molecular thinking for nanoplasmonic design, ACS Nano 6 (2012) 3655-3662, http://dx.doi.org/ 10.1021/nn301390s.

[27] D.M. Pelt, K.J. Batenburg, Fast tomographic reconstruction from limited data 
using artificial neural networks, IEEE Trans. Image Process. 22 (2013) 5238-5251, http://dx.doi.org/10.1109/TIP.2013.2283142.

[28] P. Ercius, M. Weyland, D.A. Muller, L.M. Gignac, Three-dimensional imaging of nanovoids in copper interconnects using incoherent bright field tomography, Appl. Phys. Lett. 88 (2006) 243116, http://dx.doi.org/10.1063/1.2213185.

[29] R.A. Crowther, D.J. DeRosier, A. Klug, The reconstruction of a three-dimensional structure from projections and its application to electron microscopy, Proc. R. Soc. A: Math. Phys. Eng. Sci. 317 (1970) 319-340, http://dx.doi.org/ 10.1098/rspa.1970.0119.

[30] P. Gilbert, Iterative methods for the three-dimensional reconstruction of an object from projections, J. Theor. Biol. 36 (1972) 105-117, http://dx.doi.org/ 10.1016/0022-5193(72)90180-4.

[31] H. Heidari Mezerji, W. Van den Broek, S. Bals, A practical method to determine the effective resolution in incoherent experimental electron tomography, Ultramicroscopy 111 (2011) 330-336, http://dx.doi.org/10.1016/j. ultramic.2011.01.021.
[32] K.J. Batenburg, J. Sijbers, DART: a practical reconstruction algorithm for discrete tomography, IEEE Trans. Image Process. 20 (2011) 2542-2553, http://dx. doi.org/10.1109/TIP.2011.2131661.

[33] B. Goris, W. Van den Broek, K.J. Batenburg, H. Heidari Mezerji, S. Bals, Electron tomography based on a total variation minimization reconstruction technique, Ultramicroscopy 113 (2012) 120-130, http://dx.doi.org/10.1016/j. ultramic.2011.11.004.

[34] S. Link, M.A. El-Sayed, Shape and size dependence of radiative, non-radiative and photothermal properties of gold nanocrystals, Int. Rev. Phys. Chem. 19 (2000) 409-453, http://dx.doi.org/10.1080/01442350050034180.

[35] E.M. Perassi, J.C. Hernandez-Garrido, M.S. Moreno, E.R. Encina, E.A. Coronado P.A. Midgley, Using highly accurate 3D nanometrology to model the optical properties of highly irregular nanoparticles: a powerful tool for rational design of plasmonic devices, Nano Lett. 10 (2010) 2097-2104, http://dx.doi.org/ 10.1021/nl1005492. 